\title{
Emission-line properties of MG 2016+112: A luminous obscured quasar at high redshift ${ }^{\star}$
}

\author{
T. Yamada ${ }^{1}$, S. Yamazaki ${ }^{1}$, M. Hattori ${ }^{1}$, G. Soucail ${ }^{2}$, and J.-P. Kneib ${ }^{2}$ \\ 1 Astronomical Institute, Tohoku University, Aoba-ku, Sendai 980-77, Japan \\ 2 OMP, 14 Av. E. Belin, 31400 Toulouse, France \\ Received 5 August 1999 / Accepted 27 November 2000
}

\begin{abstract}
We present new high signal-to-noise ratio spectra of the components $\mathrm{B}$ and $\mathrm{C}$ of the gravitational lensing system MG 2016+112. We show that image C displays strong emission lines of Ly $\alpha, \mathrm{NV}$, CIV, HeII, and CIII] redshifted to $z=3.27$, similar to images A and B. We examine the emission-line flux ratios in order to put constraints on the lens models as well as to investigate the intrinsic nature of MG 2016+112. The observed line ratios of $\mathrm{B}$ and $\mathrm{C}$ are consistent with those expected in the simple photo-ionization models for narrow-line region of active galactic nuclei (AGN) except for the enhanced NV lines. The line ratios difference of components B and $\mathrm{C}$ can be interpreted as a difference in ionization parameters. This result is consistent with lens model prediction that $\mathrm{C}$ is a fold image of a slightly outer part of the nucleus. MG 2016+112 is known to be very unique among the high-redshift AGN; it is neither an ordinary broad-line quasar nor a powerful radio galaxy as indicated by the width and flux ratio of the emission lines. Together with other observed properties discussed in literature, we argue that MG 2016+112 may be an obscured luminous radio-quiet quasar.
\end{abstract}

Key words. galaxies: active - galaxies: quasars: emission lines - galaxies: quasars: individual: MG 2016+112

\section{Introduction}

MG 2016+112 is one of the first discovered gravitationallens system. Lawrence et al. (1984) observed three distinct radio sources, A, B, and C. Further higher resolution radio map resolved $\mathrm{C}$ into multiple components (Garrett et al. 1994, 1996). At optical wavelength, the image A and $\mathrm{B}$ are point-sources but the image $\mathrm{C}$ is a fainter resolved object (Lawrence et al. 1984; Schneider et al. 1985, 1986). Spectroscopic observations revealed that A and B show very similar spectra dominated by strong ultra-violet (UV) emission-lines redshifted to $z=3.27$ (Lawrence et al. 1984; Schneider et al. 1987). Although the optical spectrum of $\mathrm{C}$ has not been fully published so $\mathrm{far}^{1}$, it has always been considered at the same redshift as A and B after its detection in the redshifted Ly $\alpha$ narrow-band data (Schneider et al. 1986).

The MG 2016+112 lens system is enigmatic for 3 reasons. First, the lensed object itself is very unique among known high-redshift galaxies (Lawrence et al. 1984). It has been conventionally called a "quasar" since it is

Send offprint requests to: T. Yamada,

e-mail: yamada@astr.tohoku.ac.jp

* Based on observations with the Canada-France-Hawaii Telescope at Mauna Kea, Hawaii, USA

1 Lawrence et al. (1996) show a figure of the spectrum obtained with the Keck telescope but there is no detail description. a fairly luminous point-like object. However, A and B show only narrow ultra-violet (UV) emission lines and thus MG 2016+112 is not an ordinary broad-line quasar. Although strong narrow emission lines are typically seen for high-redshift powerful radio galaxies (HzPRGs), MG 2016+112 appears somewhat different from the known HzPRGs. Indeed, while HzPRGs are typically extended in optical images and have lobe-dominated radio structures with a scale of at least a few tens of $\mathrm{kpc}, \mathrm{A}$ and B show only point-like features even in high-resolution optical and radio images. It is important to investigate the true nature of the lensed object.

Second, image $\mathrm{C}$ is a very complex object: at optical and near-infrared (NIR) wavelength, C is resolved and has an arc-like morphology. Radio-to-optical flux ratio of $\mathrm{C}$ is several times larger than those of A and B and cannot be explained by variability and time delay. At radio wavelength, $\mathrm{C}$ is resolved into two components, $\mathrm{C}_{1}$ and $\mathrm{C}_{2}$. While $\mathrm{A}, \mathrm{B}$ and $\mathrm{C}_{2}$ (sometimes referred as $\mathrm{C}^{\prime}$ in literatures) are point-like objects even at 15-mas resolution, $\mathrm{C}_{1}$ has been further resolved into three chain-like components (Garrett et al. 1996). Radio spectral shape of $\mathrm{C}_{2}$ is similar to those of $\mathrm{A}$ and $\mathrm{B}(\alpha=0.8)$ but $\mathrm{C}_{1}$ has significantly flatter one $(\alpha=0.2)$. Whether $\mathrm{C}_{1}$ is a radio galaxy at different redshift or a lensed image of the outer structure of the radio source at $z=3.27$ is still in question.

Finally the nature of the lens producing this multiple image system has been enigmatic. Deep optical and 
NIR images have detected a red galaxy D amid images A, B and C (Schneider et al. 1986; Langston et al. 1991; Lawrence et al. 1993). Galaxy D seems to correspond to an evolved giant elliptical galaxy at $z \sim 1$. However, the image separation requires a mass-to-light ratio for $\mathrm{D}$ much larger than typical one. While lens models have assumed the existence of a high-redshift massive cluster as an additional source of lensing mass (Narasimha et al. 1984, 1987, 1989; Nair \& Garrett 1997; Langston et al. 1991), at first no signature of such a cluster has been seen in the optical and NIR observations (Schneider et al. 1987; Langston et al. 1991).

Hattori et al. (1997) detected an extended X-ray emission in the direction of MG $2016+112$, possibly emitted by the hot gas in the lensing cluster of galaxy. A strong emission-line-like feature consistent with iron lines redshifted to $z \sim 1$ was detected. This discovery could in principle solve the "dark-lens" problem for MG 2016+112, but opened another question of a "dark cluster", namely, the lack of optical counterpart of the X-ray hot gas. Very recently, Soucail et al. (2000) and Kneib et al. (1997) spectroscopically detected several galaxies at $z \sim 1$ and Benítez et al. (1999) observed a possible color-magnitude sequence of faint red galaxies in this field arguing for the existence of a distant massive structure at $z \sim 1$.

We have obtained new spectra of components B, C, D of the MG $2016+112$ system. In this paper, we analyze the emission lines of image $\mathrm{B}$ and $\mathrm{C}$ in order not only to investigate the intrinsic nature of MG 2016+112 but also to understand the component $\mathrm{C}$ in view of the lens-model prediction. Observations and data reduction are described in Sect. 2. In Sect. 3, we examine the observed emission line properties. The line flux ratios are compared with the prediction of the photo-ionization models as well as those of other high-redshift objects in various categories. In Sect. 4, the nature of image $\mathrm{C}$ is discussed in the context of lens models. We then argue that MG $2016+112$ may be a radio-quiet obscured quasar based on the results obtained in Sect. 3 as well as those discussed in literatures. Throughout this paper we use $H_{0}=50 \mathrm{~km} \mathrm{~s}^{-1} / \mathrm{Mpc}$, $\Omega_{0}=1$ and $\lambda=0$.

\section{Observations and the obtained spectra}

Spectroscopic observations were made with the CanadaFrance-Hawaii Telescope (CFHT) using the Subarcsecond Imaging Spectrograph (SIS; Le Févre et al. 1994) in August 1997. The Stis2 $2048 \times 2048$ CCD with $21 \mu \mathrm{m}$ pixel and the R150 grating were used and the resultant dispersion is $2.88 \AA$ per pixel. The wavelength coverage extends from 4500 to $9000 \AA$. Two multi-slit masks were used to obtain spectra of candidate cluster galaxies in the field. Each slit has 7.8-arcsec-long and 0.78-arcsecwide aperture, which gives $\sim 18 \AA$ instrumental resolution. MG $2016+112 \mathrm{~B}, \mathrm{~B}_{1}$, and D were observed within one slit of the first mask (Mask1) and $\mathrm{C}$ with a slit in the second one (Mask2). In total, 6 and 5.8 hours exposures were obtained for Mask1 and Mask2, respectively.

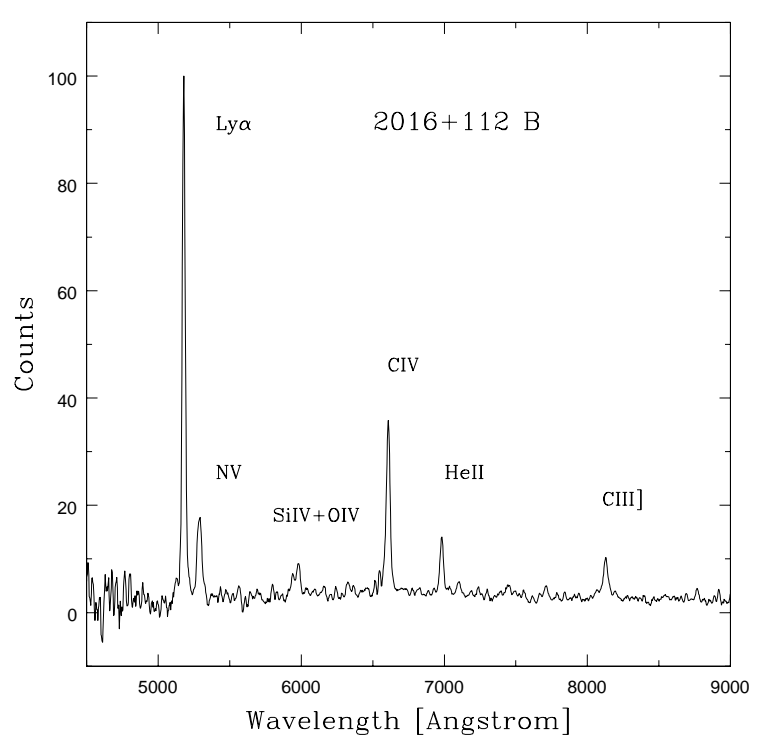

Fig. 1. Observed spectra of MG 2016+112 B

The data was pre-reduced using standard IRAF ${ }^{2}$ tasks. We then follow the reduction procedure of multi-slit data described in Le Févre et al. (1995). Wavelength calibration was done with the arc-line spectra taken at the observation. Typical internal error of wavelength determination is $\sim 0.3 \AA$. The flux calibration was done using the spectroscopic standard star GD248 (Oke 1990). Note that the flux calibration becomes more uncertain above 7500-8000 because no order-separating filter was used and the flux is contaminated by the UV and blue contribution of the second order filter. We tried to carefully take into account this effect but flux calibration may remain uncertain above $8000 \AA$.

Figures 1 and 2 show the obtained spectra of image $\mathrm{B}$ and $\mathrm{C}$ smoothed with a 5 pixel Gaussian filter. Strong UV emission lines of Ly $\alpha$, NV $\lambda \lambda 1240 \AA$, CIV $\lambda \lambda 1549 \AA$, HeII $\lambda 1640 \AA$, and CIII] $\lambda 1909 \AA$ are seen in both of the spectra. SiIV and OV lines at $1400 \AA$ are clearly seen in the $\mathrm{B}$ spectrum but only marginal in $\mathrm{C}$. The redshift of $\mathrm{C}$ is the same as B within the uncertainties. There is no sign of contamination by a system at a different redshift.

\section{Emission-line properties of MG $2016+112$ $B$ and $C$}

We now describe the observed properties of the emission lines of image B and C. The velocity width (FWHM) corrected for instrumental resolution and the relative flux values measured by Gaussian fitting procedure are listed in Tables 1 and 2, respectively. The flux values are normalized to CIV lines. We corrected for the reddening by the Galaxy using the value of the extinction in this region, $A_{V}=0.67$ and $A_{I}=0.36$, derived by Benítez et al. (1999). No correction for the internal reddening was applied since the rest-frame wavelength of the emission lines

${ }^{2}$ IRAF is distributed by NOAO, which are operated by AURA, Inc., under cooperative agreement with NSF. 
Table 1. Line width ${ }^{\mathrm{a}}$

\begin{tabular}{lccccc}
\hline & MG $2016+112 \mathrm{~A}^{\mathrm{b}}$ & MG 2016+112 B & MG 2016+112 C & F10214 & SMM 02399 $^{\mathrm{d}}$ \\
\hline Ly $\alpha$ & $<1000$ & 460 & 630 & 900 & 1850 \\
$\mathrm{NV}$ & $<1600$ & 880 & 840 & 1700 & 1790 \\
$\mathrm{CIV}$ & $<1100$ & 580 & 580 & 1200 & 1560 \\
$\mathrm{HeII}$ & $<1200$ & 460 & 440 & 1150 & 2800 \\
$\mathrm{CIII}]$ & - & 750 & 1100 & & 7700 \\
$\mathrm{CIII}$ narrow & - & 360 & 380 & 1000 & \\
CIII] broad & - & 1750 & 2550 & 3700 & \\
\hline
\end{tabular}

(a) FWHM in $\mathrm{km} \mathrm{s}^{-1}$.

(b) From Lawrence et al. (1984).

(c) From Searjeant et al. (1998).

(d) From Ivison et al. (1998).

Table 2. Relative flux of the emission lines ${ }^{\mathrm{a}}$

\begin{tabular}{|c|c|c|c|c|c|c|c|c|c|}
\hline & MG & $2016+112$ B & MG & $2016+112 \mathrm{C}$ & F10214 & SMM 02399 & $\mathrm{HzPRG}^{\mathrm{d}}$ & Quasar BLR $^{\mathrm{d}}$ & Seyfert $2^{\mathrm{d}}$ \\
\hline $\operatorname{Ly} \alpha$ & 2.73 & $(0.09)$ & 3.81 & $(0.43)$ & 0.53 & 3.39 & 2.17 & 8.52 & 4.52 \\
\hline NV & 0.66 & $(0.07)$ & 1.19 & $(0.48)$ & 0.74 & 2.20 & 0.57 & 0.42 & \\
\hline CIV & 1.00 & & 1.00 & & 1.00 & 1.00 & 1.00 & 1.00 & 1.00 \\
\hline HeII & 0.28 & $(0.03)$ & 0.55 & $(0.10)$ & 0.53 & 0.36 & 0.12 & 0.87 & 0.17 \\
\hline CIII] & 0.40 & $(0.04)$ & 1.32 & $(0.27)$ & & 2.70 & 0.45 & 0.49 & 0.46 \\
\hline CIII] narrow & 0.17 & $(0.02)$ & 0.51 & $(0.10)$ & 0.34 & & & & \\
\hline CIII] broad & 0.29 & $(0.06)$ & 1.25 & $(0.51)$ & 0.28 & & & & \\
\hline
\end{tabular}

(a) Normalized to CIV line. Numbers in parenthesis show the error values.

(b) From Searjeant et al. (1998).

(c) Evaluated by us from the values of equivalent width in Ivison et al. (1998).

(d) From McCarthy et al. (1993).

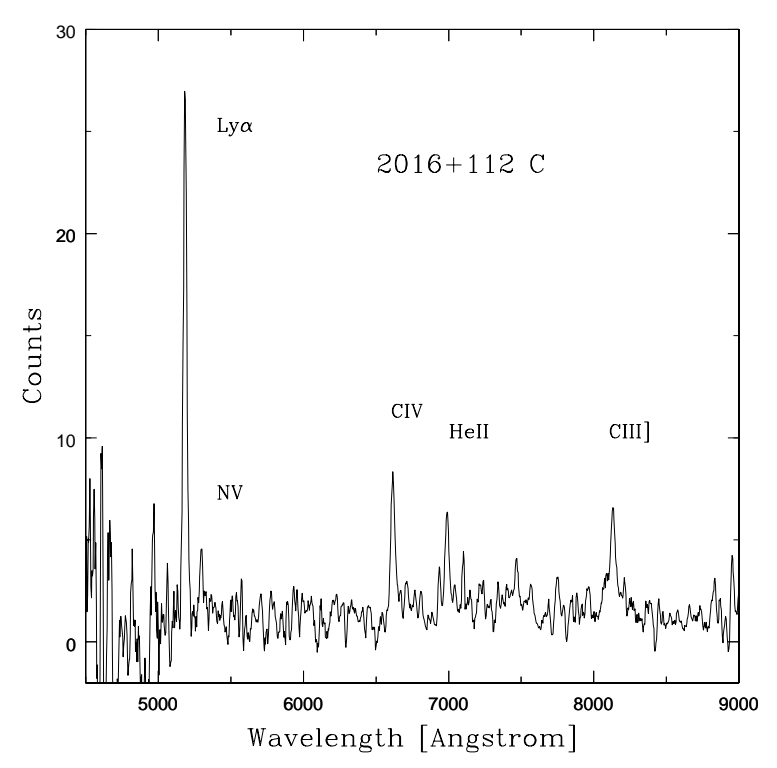

Fig. 2. Observed spectra of MG 2016+112 C

concerned here are rather close. We treat the unresolved doublet lines as a single line.

The velocity width of the lines are between 450-900 $\mathrm{kms}^{-1}$. These lines are much narrower than quasar broad lines which have typical width of 5000-10000 $\mathrm{kms}^{-1}$ but as narrow as those of HzPRG, 500-1000 km s${ }^{-1}$ (e.g., McCarthy 1993). In Table 1, we compare the emission-line width to the two infraredselected gravitationally-lensed type-2 AGN at high redshift, namely IRAS F10214+4724 (e.g., Rowan-Robinson et al. 1991) and SMM 02399-0136 (Ivison et al. 1998).

CIII] line of both $\mathrm{B}$ and $\mathrm{C}$ seem to have broad wings. Figures 3a,b shows the results of the two-component Gaussian fitting of the CIII] lines for B and $\mathrm{C}$ and $3 \mathrm{c}$ and $3 \mathrm{~d}$ shows those with single-component for comparison. They cannot be perfectly fitted with a single component but can be better fitted with a narrow plus broad components as shown in the figures. It seems strange that the CIII] line has a broad component while the CIV line, which comes from the gas at higher ionization stage and may be closer to the central engine, shows only a narrow one. It may be due to the extinction of the broad component of the CIV line. Another possibility is that the wings are the lines of other ions contaminating this spectral range.

The broad CIII] feature is not a unique characteristics of MG 2016+112 lensed object. IRAS F10214+4724 (Searjeant et al. 1997) and SMM 02399-0136 (Ivison et al. 1998) also show similar property. Searjeant et al. (1997) 
considered the possibility of contamination by SiIII] lines to the blue-wing feature of the CIII] line in the spectrum of IRAS F10214+4724 but the model cannot fully explain the observed feature (Searjeant et al. 1997). For the case of MG 2016+112, the broad component seems to extend both side of the narrow component (Figs. 3a,b) and thus it is also difficult to be explained by the contamination of SiIII] lines. It is interesting that those two gravitationallylensed type-2 AGN as well as MG 2016+112 show evidence of a broad CIII] line.

Next, we investigate the line flux ratios. There are significant differences between line ratios of component $\mathrm{B}$ and $\mathrm{C}$. While the HeII/CIV ratio of image $\mathrm{B}$ is 0.28 , the ratio of $\mathrm{C}$ is 0.55 . Also, the $\mathrm{CIII}] / \mathrm{CIV}$ ratio of $\mathrm{B}$ and $\mathrm{C}$ is 0.17 and 0.51 , respectively if we consider the narrow components. If we fit the lines with single Gaussian component, then the ratio is 0.40 and 1.32 . The emission-line gas in image $\mathrm{B}$ seems to be at higher ionization stage than in C. In Fig 4, we compare these line ratios with those predicted by photoionization models (CLOUDY90, Ferland 1988). We examined the cases with hydrogen density $n_{\mathrm{H}}=100$ and $1000 \mathrm{~cm}^{-3}$, power-low ionization continuum with $\alpha=1$ and 1.4 , and a range of ionization parameter, $\Gamma=10^{-2.5}-10^{-1}$ and solar-abundance. The obtained line ratios are fairly consistent with the typical photo-ionization models considered for narrow-line region of AGN. The differences in line ratios between $\mathrm{B}$ and $\mathrm{C}$ may be interpreted as the difference in ionization parameters.

There may be an effect of the reddening by dust in the object on the observed line ratio. According to Calzetti's reddening formula for starburst galaxies (Sawicki \& Yee 1998), the reddening of $E(B-V)=1$ shifts the observed values with $\Delta \log (\mathrm{CIV} / \mathrm{HeII})=-0.12$ and $\Delta \log (\mathrm{CIII}] / \mathrm{HeII})=+0.30$, respectively, which is shown by the arrow in Fig. 4. Even if there is a fairly large amount of reddening, $E(B-V) \sim 1$, the discussion we give here is not so much affected.

There are weak but fairly significant NV lines in the spectra of B and C. In Fig. 5 we plotted the observed NV line ratios as well as those predicted by photoionization models. Clearly, photoionizatin models that can explain the line ratios at lower ionization state are not consistent with the observed NV flux. The observed NV lines are more than several times stronger than the predicted value. Reddening correction moves the points further away from the models. The origin of NV line may be different from those of other lines since the ionization potential of $\mathrm{N}^{++++}$is $77.5 \mathrm{eV}$ which is significantly higher than those of CIV $(47.9 \mathrm{eV}), \mathrm{CIII}](24.4 \mathrm{eV})$, and HeII $(24.6 \mathrm{eV})$. Indeed, the observed line width of the NV line is larger than others (see Table 1). The enhancement of the NV line may also be due to the nitrogen over abundance. The excess of the NV line intensity is not special for MG 2016+112 B; Hamman \& Ferland (1993) also compare their photoionization models with the observed lines of broad-line region (BLR) of quasars and found a large nitrogen over abundance. There is also similar nitrogen problem for the optical NII lines observed in the spectra of local AGN (e.g., Osterbrook 1986).

The fast shock models (Mouri \& Taniguchi, private communication) can provide the NV/CIII] and NV/HeII ratios that match the observed values but then predict too strong CIV lines. The line ratios observed in image B and $\mathrm{C}$ are not perfectly understood with simple photoionization or shock models.

In Fig. 6, we also plot typical line ratios of various types of high-redshift AGN taken from McCarthy (1993), ultra-steep spectrum HzPRGs compiled by Röettgering et al. (1997), and the infrared-selected type-2 quasars, IRAS F10214+4724 (Serjeant et al. 1997). Many of the HzPRGs in Röettgering et al. (1997) are distributed at around $\log (\mathrm{CIV} / \mathrm{HeII}) \sim 0.2$ and $\log (\mathrm{CIII}] / \mathrm{HeII}) \sim-0.2$, which is consistent with the average flux ratio given by McCarthy (1993). The line ratios of image B is very different from typical HzPRGs. The line ratios of $\mathrm{C}$ and IRAS F10214+4724 lies near the edge of the distribution of line ratios of HzPRGs.

We also plotted the line ratio of the broad lines observed in quasar spectra compiled by Baldwin et al. (1979). They are very different from both HzPRGs and image $\mathrm{B}$ and $\mathrm{C}$ and can be explained by the photoionization models with high density such as $10^{9-10} \mathrm{~cm}^{-3}$.

\section{Discussion}

\subsection{Lens models}

Several lens models have been proposed for MG 2016+112 (Narasimha et al. 1984, 1987, 1989; Langston et al. 1991; Nair \& Garrett 1997; Benítez et al. 1999). These models are constructed to match the optical and/or radio data such as image positions and flux ratios of the various components. The results of our spectroscopic observations can be used to put further constraints on the lens model.

As discussed in Sect. 3, the difference of CIII], CIV, and HeII line ratios in image B and $\mathrm{C}$ can be interpreted as a difference in ionization degree. Photoionization model predict smaller ionization parameter for $\mathrm{C}$, which is a natural consequence if the component $\mathrm{C}$ is dominated by the light of a region at $\sim 1.5-2$ times larger radius from the nucleus than the source region of the component B (assuming similar density).

It is unlikely that the differences in line ratios of $\mathrm{B}$ and $\mathrm{C}$ is due to the contamination by a possible radio galaxy at the similar redshift assumed as the counterpart of the bright flat-spectrum radio component $\mathrm{C}_{1}$. If the high CIII]/CIV ratio observed in the spectrum of image $\mathrm{C}$ is due to such a contamination, for example, the assumed radio galaxy should have CIII]/CIV ratio larger than $\sim 1$. Radio galaxies rarely show such high CIII]/CIV ratio.

Our spectroscopic results thus support lens models like the ones proposed by Langston et al. (1991) and Benítez et al. (1999). In these models, the AGN and the narrowemission-line region is located somewhat outside the fold caustic in the source plane, which results in forming the 

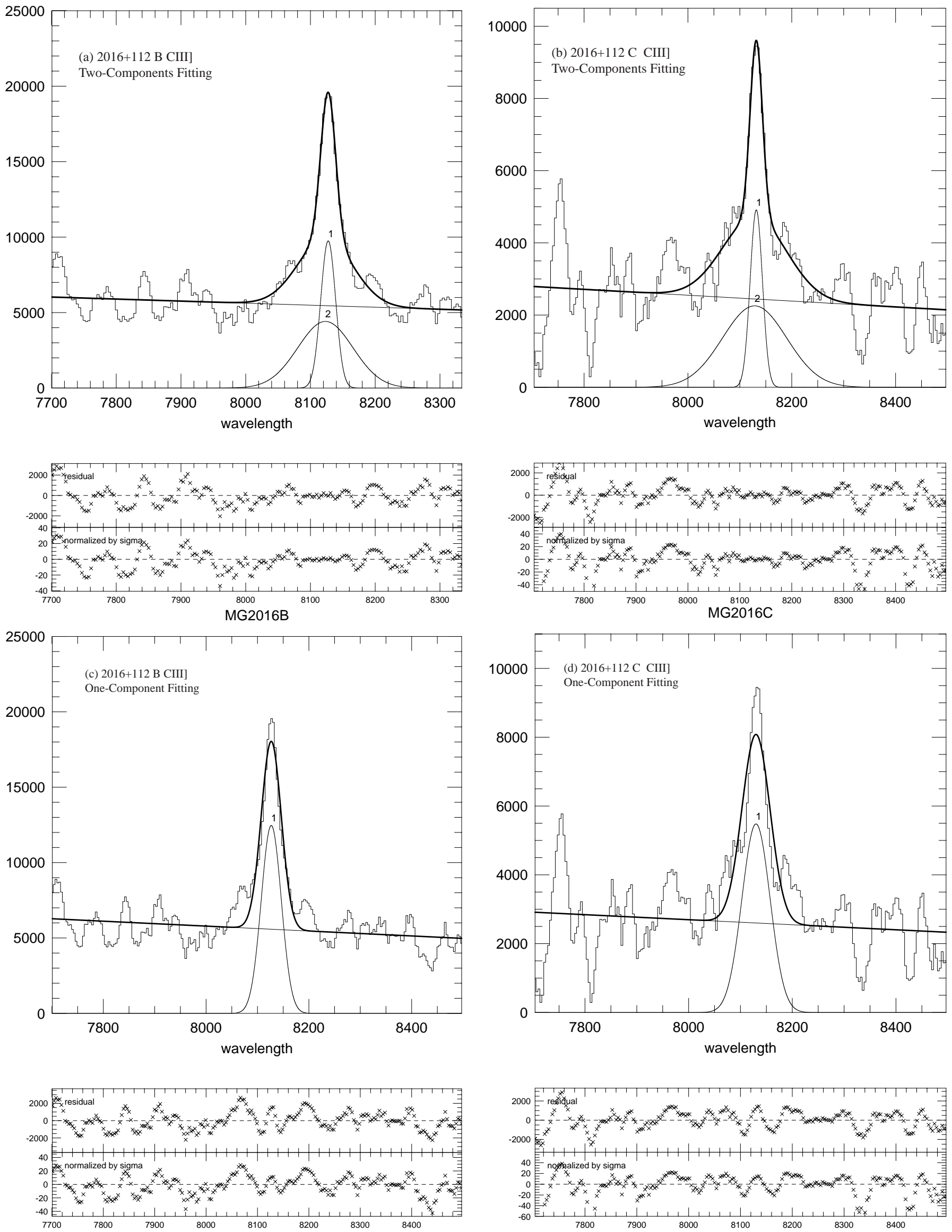

Fig. 3. Results of the two-components Gaussian fitting for the CIII] lines of component B (panel a)) and C (panel b)) and of the one-component ones (panels $\mathbf{c}$ ) and $\mathbf{d}$ )). The LDECONV task developed by Michitoshi Yoshida was used for the fitting 


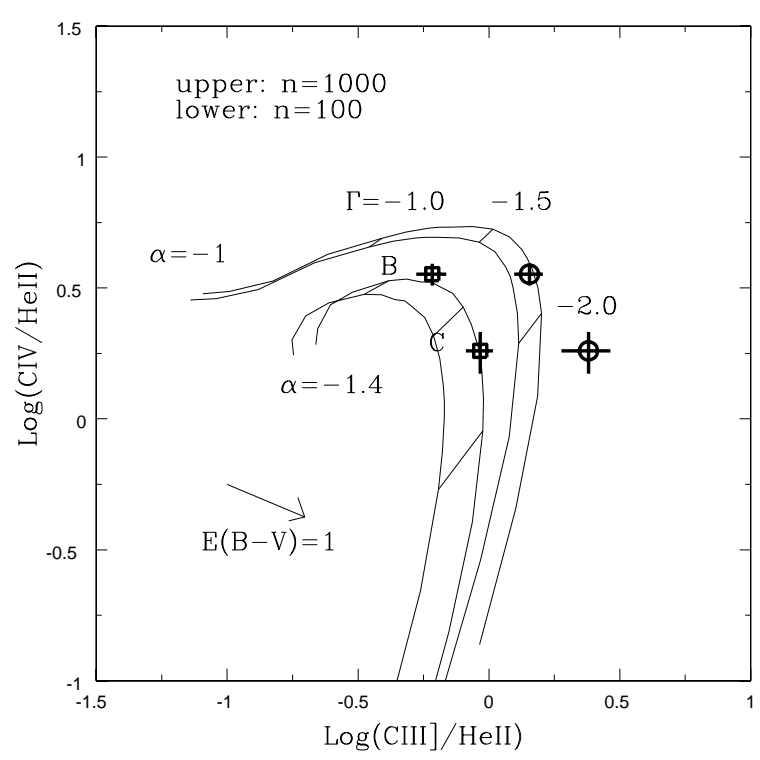

Fig. 4. Flux ratios of the emission lines seen in the spectrum of image B and C. Squares show those using only the narrow component of the CIII] line and circles those using the CIII] flux obtained with single-component fitting. The grids show the line flux ratio predicted by photo-ionization models calculated using CLOUDY90 (Ferland et al. 1988). The cases of hydrogen density of $100 \mathrm{~cm}^{-3}$ and $1000 \mathrm{~cm}^{-3}$ and input power-law spectra with energy index -1.0 and -1.4 are plotted for ranges of ionization parameter $\log \Gamma=-1.0$ to -2.5 (solid lines). The arrow shows the effect of the reddening calculated with the Calzetti's relation for starburst galaxies. 4 and $E(B-V)=1$

images A and B. The outer regions of the source extends over the caustic and the region which is very close to the caustic is largely amplified and form an arc-like lensed images at the position of component $\mathrm{C}$. Radio sources at position C are interpreted as a lensed image of a "jet" which extends inside the caustic. The counter images of the component $\mathrm{C}$ will exist at position $\mathrm{A}$ and $\mathrm{B}$ but is mostly swamped by the AGN light due to a smaller amplification factor (compared to position $\mathrm{C}$ ).

\subsection{MG 2016+112: An obscured luminous radio-quiet quasar?}

\subsubsection{Intrinsic power of MG $2016+112$}

The observed radio flux density of image $\mathrm{B}$ at $1.47 \mathrm{GHz}$ is $61.7 \mathrm{mJy}$ (Lawrence et al. 1984), which corresponds to a luminosity density $L_{2.7 \mathrm{GHz}}=2.310^{34} \mathrm{erg} \mathrm{s}^{-1} \mathrm{~Hz}^{-1}$ $A_{\mathrm{GL}}^{-1}$ and $L_{8.4 \mathrm{GHz}}=9.110^{33} \mathrm{erg} \mathrm{s}^{-1} \mathrm{~Hz}^{-1} A_{\mathrm{GL}}^{-1}$ at 2.7 and $8.4 \mathrm{GHz}$, respectively, with a luminosity distance of $26.5 \mathrm{Gpc}$ and a spectral index $\alpha=-0.81$ (Lawrence et al. 1984). $A_{\mathrm{GL}}$ is a gravitational lensing amplification factor. According to the recent lens model by Benítez et al. (1999), the amplification factor of image B is estimated to be $\sim 6$.

The radio power of $\mathrm{MG} 2016+112$ is fairly large even corrected from the lensing amplification (e.g., Lawrence et al. 1984). Danese et al. (1987) obtained radio

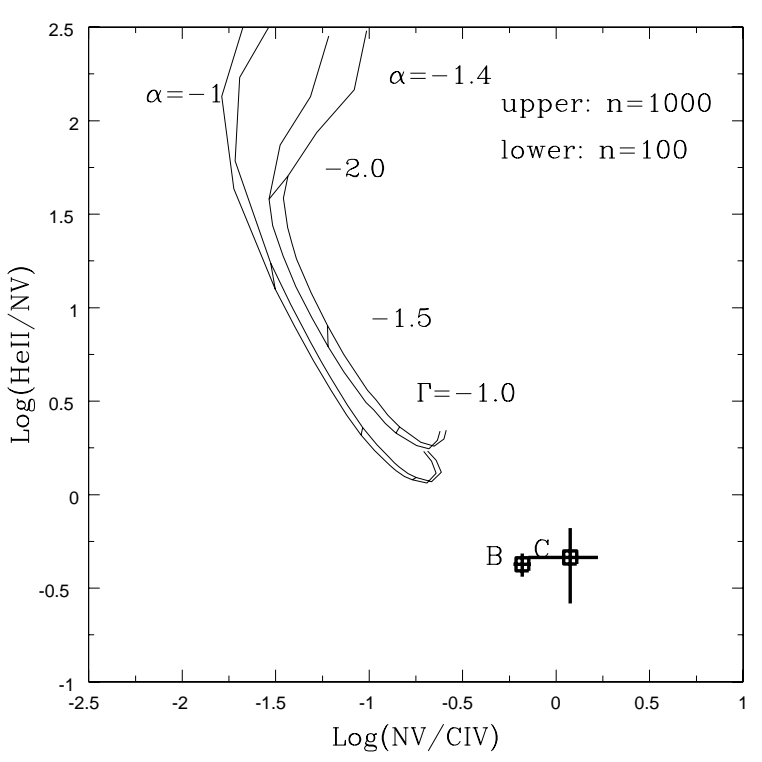

Fig. 5. Same as Fig. 4, but for NV line

luminosity functions of at $2.4 \mathrm{GHz}$ for various type of objects at $z \sim 0$. Even the most luminous local objects have radio power $\sim 10^{32} \mathrm{erg} \mathrm{s}^{-1} \mathrm{~Hz}^{-1}$, which is more than a order of magnitude fainter than MG 2016+112. Dunlop \& Peacock (1990) evaluated radio luminosity function of the radio-selected quasars and radio galaxies. Image B has radio power as large as those of very luminous radio sources at $z \sim 0.5$ which are definitely categorized as "quasars" or "powerful radio galaxies" $\left(10^{33}-10^{34} \mathrm{erg} \mathrm{s}^{-1} \mathrm{~Hz}^{-1}\right.$ at $2.7 \mathrm{GHz}$ ). Thus it is very certain that the object harbors AGN as powerful as luminous as quasars.

Bischef \& Becker (1998) investigated radio emissions for a sample of 4079 known quasars based on the NVSS radio catalog. These quasars are selected from the VerónCetty \& Verón (VCV) catalog and constitute the largest compilation so far to study the radio properties of radioand optically-selected quasars based on homogeneous radio observations in one frequency. In their Fig. 4, they presented a distribution of radio power at $8.4 \mathrm{GHz}$ with redshift. The bimodal distribution of the radio power is evident at least to $z \sim 2.5$. At higher redshift, the number of known radio-luminous quasars is too small to draw firm conclusion, but the tendency seems to hold. The radio power of MG $2016+112$ at $8.4 \mathrm{GHz}$ is likely to lie on the extension of the lower sequence, if we adopt an amplification factor of $\sim 10$. In Fig 7 we plot the observed and amplification-corrected radio power of MG 2016+112 B superposed on the figure of Bischef \& Becker's (their Fig. 4).

\subsubsection{Radio loudness}

Radio loudness of AGN is conventionally defined by the radio to optical (or ultra violet) flux ratio. Bischef \& Becker (1998) also obtained the distribution of the radio loudness for VCV quasars. According to their definition, radio-loud quasars have log $\left(L_{8.4 \mathrm{GHz}} / L_{B}\right)$ larger than 1 . 


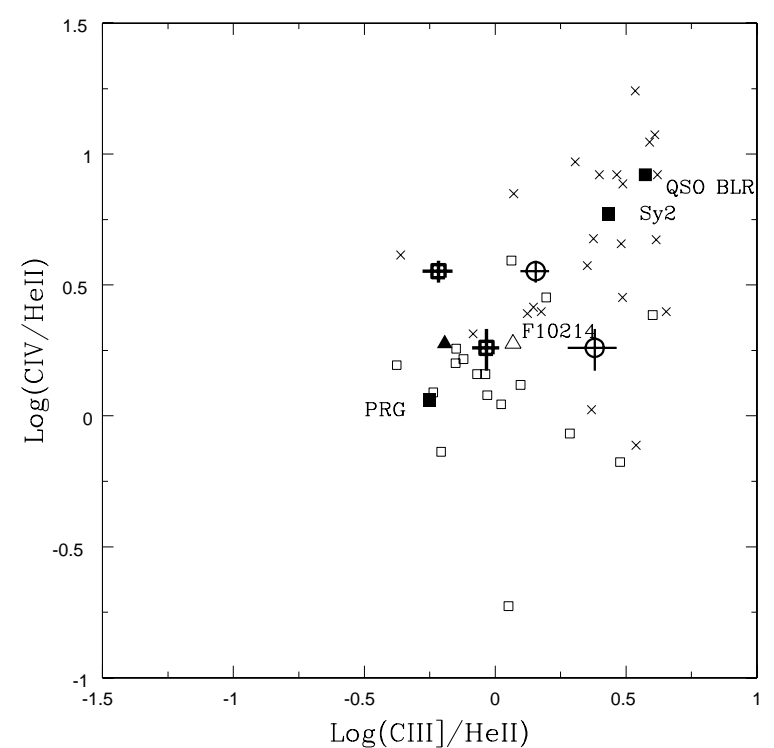

Fig. 6. Line ratios for various kinds of high-redshift AGN. The solid squares are the average line ratios given in McCarthy (1993) and the open squares are those of USS HzPRGs in Röttgering et al. (1997). Triangles are the infrared-selected type-2 AGN, IRAS F10214+4724, using the narrow-component (filled) and total (open) CIII] flux. The ratios of broad lines quasar quoted from Baldwin (1979) are shown as crosses

The upper sequence in Fig. 4 of Bischef \& Becker (1998) corresponds to radio-loud quasars and the lower to radioquiet quasars. It is difficult, however, to evaluate the radio loudness of MG $2016+112$ by using the radio to optical flux ratio since it is an obscured narrow-line object. We may see only a scattered light of the nucleus in the optical wavelength and the contamination by light from the host galaxy must be relatively large. The nominal value, $\log L_{8.4 \mathrm{GHz}} / L_{B}=3.4$, thus does not mean that this object is a radio-loud AGN. Indeed, as mentioned in the previous subsection, the observed radio power of MG 2016+112 after lensing amplification correction seems too faint to be classified as radio-loud, which suggests that this object is a radio-quiet AGN. It is not surprising that the scattered component is more than hundred times smaller than the intrinsic luminosity and the intrinsic $\log L_{8.4 \mathrm{GHz}} / L_{B}$ value of the object can be $\lesssim 1$.

How typical the radio power is if MG 2016+112 is a radio-quiet AGN? Figure 7 suggests that it may be one of the most radio active object among the radioquiet quasars at this redshift. Kukula et al. (1998) recently investigated the correlation between radio and optical luminosity of a sample of nearby radio-quiet quasars and Seyfert galaxies $(z<0.2)$. The most luminous nearby quasars with $M_{V} \sim-26$ have $\log L_{8.4 \mathrm{GHz}} \sim$ $10^{31} \mathrm{erg} \mathrm{s}^{-1} \mathrm{~Hz}^{-1}$. If the correlation holds for high-redshift quasars which are typically 50-100 times more luminous than nearby AGNs, MG $2016+112\left(L_{8.4 \mathrm{GHz}} \sim 10^{33} \mathrm{erg}\right.$ $\mathrm{s}^{-1} \mathrm{~Hz}^{-1}$ ) may be one of the most luminous quasars in optical wavelength, too, even if there is some radio excess.

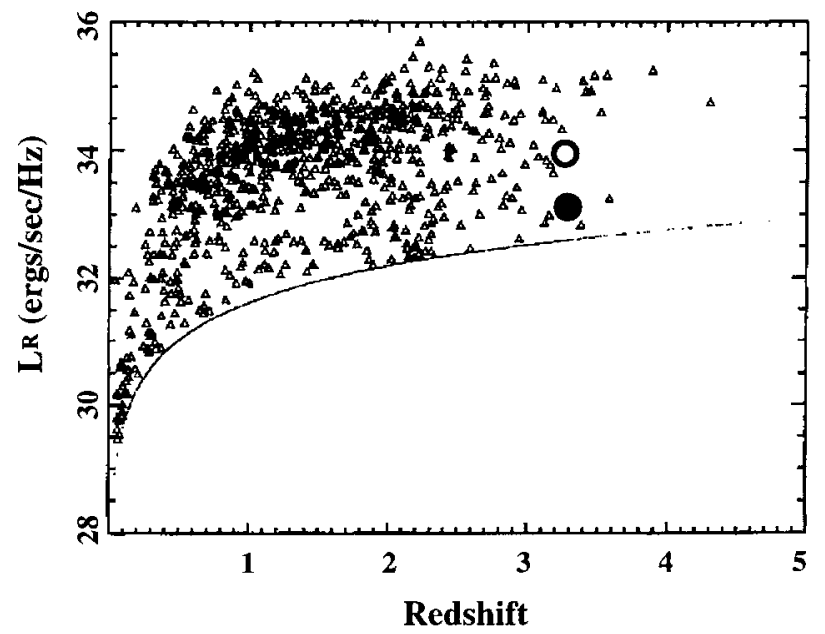

Fig. 7. The observed (thick open circle) and the lensing amplification corrected (filled circle) radio luminosities of MG 2016+112 superposed in Fig. 4 of Bischof \& Becker (1990) (scanned by us) which shows the distribution of radio luminosity at $8.4 \mathrm{GHz}$ vs redshift for VCV quasars. Crosses are values for the broad lines of type-1 quasars

\subsubsection{Morphologies}

HST NICMOS observations (Falco et al.) revealed that the rest-frame optical light of MG 2016+112 A and B is dominated by the point sources. The resolution limit of NICMOS is $\sim 0.15$ arcsec. If we adopt the lens model of Benítez et al. (1999), the size of the unresolved lensed object must be smaller than 0.03 arcsec, which corresponds to $\sim 200 \mathrm{pc}$ at $z=3.27$. It may not be surprising that $\mathrm{A}$ and $\mathrm{B}$ are not resolved if we observe only the obscured nucleus or its scattered light which may come from inside the narrow-line region.

Many of HzPRGs show resolved faint extension in NIR images which may be star lights of their host galaxies. On the other hand, the known type-2 AGN or obscured quasars at $z \sim 1-2.5$ are not resolved or only marginally resolved (Ohta et al. 1995; Almani et al. 1995; Ivison et al. 1998). There could be some difference between observable properties of host galaxies of HzPRGs and radio-quiet type-2 AGN or obscured quasars.

The radio images of $\mathrm{A}$ and $\mathrm{B}$ are also point-like with $\sim 15$ mas resolution (Garrett et al. 1996). 15 mas corresponds to about $40 \mathrm{pc}$ in the physical scale at $z=3.27$. Kukula et al. (1998) presented the $1.4 \mathrm{GHz}$ maps of the nearby radio-quiet quasars with $\sim 0.5$ arcsec resolution. They found a significant fraction of the radio emission in radio-quiet quasars originates in a compact nuclear source directly associated with the quasar. Therefore the pointlike morphology of the radio emission of MG 2016+112 is not surprising if it is a radio-quiet quasar. At the same time, some outer structures are also seen in the radio maps of the nearby radio-quiet quasars. The maximum extent of the radio emission of resolved sources is typically a few kpc. It is possible that such a structure associated with the lensed object extends over the diamond caustic of the 
source plane to form the strongly amplified radio image at the position of component $\mathrm{C}$.

\subsubsection{Summary of the possible type-2 quasar nature of MG $2016+112$}

Since only the narrow emission lines are observed in UV wavelength, MG $2016+112$ is very likely to be an obscured luminous AGN. Although it is need to see the properties of lines in the rest-frame optical wavelength redshifted to near infrared before concluding whether it is really a luminous analogue of the Seyfert 2 galaxies (namely, type- 2 quasars) or just a partially-obscured normal quasars, it is interesting to summarize the possible type- 2 quasar nature of MG 2016+112 since the number of known radioquiet luminous type- 2 quasars and candidates are still very limited. Only several examples of type-2 AGN or candidates at high redshift which are serendipitously discovered in far-infrared, sub-mm, and X-ray source surveys. If $\mathrm{MG} 2016+112$ at $z=3.27$ is really a type- 2 quasar, it is the highest-redshift one identified so far and thus provides a unique and important example for further studies of type- 2 quasars. The properties of MG $20116+112$ are summarized as follows.

(1) The central engine of MG $2016+112$ must be obscured since only the narrow emission lines are observed. (2) The radio and optical morphologies are both compact, which is not compatible with typical properties of HzPRGs but common for radio-quiet AGNs. (3) The radio power is much smaller than typical radio-loud quasars at $z \sim 3$ but consistent with luminous radio-quiet quasar. (4) Finally, the emission line flux ratios of image $\mathrm{B}$ are not compatible with typical HzPRGs.

We thus consider that MG $2016+112$ is not a typical powerful radio galaxies but may be naturally classified as a rare example of a high-redshift radio-quiet type- 2 quasar.

Since IRAS F10214+4724 and SMM 02399-0136 are detected in CO in sub-millimeter (Rowan-Robinson et al. 1993; Ivison et al. 1998; Frayer et al. 1998), it will be interesting to investigate the cold gas and dust properties of MG 2016+112. As expected from the obscuration of the nucleus, large dust content may be a general property of type-2 quasars.

Acknowledgements. We thank Drs. H. Mouri and Y. Taniguchi for kindly providing us the results of the shock-model calculations. This research was partially supported by grants-in-aid for scientific research of the Japanese Ministry of Education, Science, Sports and Culture (09740168, 07055044). JPK thanks CNRS for support and the Yamada Science Foundation for fruitful visits in Japan. MH also thanks the financial supports of Yamada Science Foundation.

\section{References}

Almaini, O., Boyle, B. J., Griffiths, R. E., et al. 1995, MNRAS, 277, L31

Baldwin, J. A. 1979, Active Galactic Nuclei (A79-50785 22-90) (Cambridge, Cambridge University Press), 51
Benítez, N., Broadhurst, T., Rosati, P., et al. 1999, ApJ, 527, 31

Bischof, O. B., \& Becker, R. H. 1997, AJ, 113, 2000

Danese, L., Franceschini, A., Toffolatti, L., \& De Zotti, G. 1987, ApJL, 318, L15

Dunlop, J. S., \& Peacock, J. A. 1990, MNRAS, 247, 19

Ferland, G. J., Korista, K. T., Verner, D. A., et al. 1998, Publ. ASP Inv. Rev., 110

Frayer, D. T., Ivison, R. J., Scoville, N. Z., et al. 1998, ApJL, 506, L7

Garrett, M. A., Muxlow, T. W. B., Patnaik, A. R., \& Walsh, D. 1994, MNRAS, 269, 902

Garrett, M. A., Porcas, R. W., Nair, S., \& Patnaik, A. R. 1996, MNRAS, 279, L7

Hamann, F., \& Ferland, G. 1993, ApJ, 418, 11

Hattori, M., et al. 1997, Nature, 388, 146

Ivison, R. J., Smail, I., Le Borgne, J.-F., et al. 1998, MNRAS, 298, 583

Kneib, J.-P., Soucail, G., Jaunsen, A., et al. 1998, CFHT Information Bulletin, No. 38

Kukula, M. J., Dunlop, J. S., Hughes, D. H., \& Rawlings, S. 1998, MNRAS, 297, 366

Langston, G., Fischer, J., \& Aspin, C. 1991, AJ, 102, 1253

Lawrence, C. R. 1996, IAU Symp., 173, 299

Lawrence, C. R., Schneider, D. P., Schmidet, M., et al. 1984, Science, 223, 46

Lawrence, C. R., Neugebauer, G., \& Matthews, K. 1993, AJ, 105,17

Le Févre, O., Crampton, D., Felenbok, P., \& Monnet, G. 1994, A\&A, 282, 325

Le Févre, O., Crampton, D., Lilly, S. J., Hammer, F., \& Tresse, L. 1995, ApJ, 455, 60

McCarthy, P. J. 1993, ARA\&A, 31, 639

Nair, S., \& Garrett, M. A. 1997, MNRAS, 284, 58

Narasimha, D., Subramanian, K., \& Chitre, S. M. 1984, ApJ, 283,512

Narasimha, D., Subramanian, K., \& Chitre, S. M. 1987, ApJ, 315,434

Narasimha, D., \& Chitre, S. M. 1989, AJ, 97, 327

Ohta, K., Yamada, T., Nakanishi, K., et al. 1996, ApJL, 458, L57

Oke, J. B. 1990, AJ, 99, 1621

Osterbrock, D. E. 1986, Astrophysics of Gaseous Nebulae and Active Galactic Nuclei (Mill Valley; University Science Books)

Röettgering, H. J. A., Van Ojik, R., Miley, G. K., et al. 1997, A\&A, 326, 505

Rowan-Robinson, M., et al. 1991, Nature, 351, 719

Rowan-Robinson, M., et al. 1993, MNRAS, 261, 513

Sawicki, M., \& Yee, H. K. C. 1998, AJ, 115, 1329

Schneider, D. P., Lawrence, C. R., Schmidt, M., et al. 1985, ApJ, 294, 66

Schneider, D. P., Gunn, J. E., Turner, E. L., et al. 1986, AJ, 91, 991

Schneider, D. P., Gunn, J. E., Turner, E. L., et al. 1987, AJ, 94,12

Serjeant, S., Rawlings, S., Lacy, M., et al. 1998, MNRAS, 298, 321

Soucail, G., Kneib, J.-P., Jaunsen, A., et al. 2000, A\&A, submitted [astro-ph/0006382]

Tresse, L., Rola, C., Hammer, F., et al. 1996, MNRAS, 281, 847

Veron-Cetty, M. P., \& Veron, P. 1996, European Southern Observatory Scientific Report, 17, 1 